\title{
A rapid review investigating the potential impact of a pandemic on the mental health of young people aged 12-25 years
}

\author{
A. O'Reilly $^{1}$ (1) , M. Tibbs ${ }^{1}$, A. Booth ${ }^{1}$, E. Doyle ${ }^{1}$, B. McKeague ${ }^{2}$ and J. Moore ${ }^{1}$ \\ 1 Jigsaw - The National Centre for Youth Mental Health, Dublin, Ireland \\ 2 Department of Psychology, Maynooth University, Maynooth, County Kildare
}

Objectives: In March 2020, the World Health Organization (WHO) officially declared the spread of coronavirus disease 2019 (COVID-19) as a pandemic. Adolescence and early adulthood are peak times for the onset of mental health difficulties. Exposure to a pandemic during this vulnerable developmental period places young people at significant risk of negative psychological experiences. The objective of this research was to summarise existing evidence on the potential impact of a pandemic on the mental health of 12-25 year olds.

Methods: A rapid review of the published peer-reviewed literature, published between 1985 and 2020, using PsycINFO (Proquest) and Medline (Proquest) was conducted. Narrative synthesis was used across studies to identify key themes and concepts.

Results: This review found 3,359 papers, which was reduced to 12 papers for data extraction. Results regarding the prevalence of psychological difficulties in youth were mixed, with some studies finding this group experience heightened distress during an infectious disease outbreak, and others finding no age differences or higher distress among adults. Gender, coping, self-reported physical health and adoption of precautionary measures appear to play a role in moderating the psychological impact of an infectious disease outbreak. Most studies were conducted after the peak of an epidemic/pandemic or in the recovery period.

Conclusions: More longitudinal research with young people, particularly adolescents in the general population, before and during the early stages of an infectious disease outbreak is needed to obtain a clear understanding of how best to support young people during these events.

Received 15 May 2020; Revised 28 July 2020; Accepted 07 September 2020; First published online 11 September 2020

Key words: Coronavirus, COVID-19, epidemic, infectious disease outbreak, pandemic, rapid review, youth mental health.

\section{Introduction}

A pandemic is characterised by the simultaneous worldwide spread of a novel infectious disease and typically causes widespread economic, social and political disruption (Doshi, 2011; Kelly, 2011). Although infrequent, some evidence suggests that globalisation has increased the likelihood of their occurrence (Madhav et al. 2017). Individuals affected by an infectious disease outbreak, such as a pandemic, often experience increased anxiety, particularly around contracting the illness, a higher incidence of mental health difficulties and heightened feelings of helplessness and stigma (Hall et al. 2008; Douglas et al. 2009; Rubin et al. 2010; Sim et al. 2010; Kelly, 2020). Mitigating the impact of a pandemic typically requires a large-scale, coordinated public health response [World Health Organization (WHO), 2017]. Risk-based measures

Address for correspondence: Dr A. O'Reilly, Jigsaw - The National Centre for Youth Mental Health, 16 Westland Square, Pearse St., Dublin, Ireland.

(Email: Aileen.oreilly@jigsaw.ie) including social/physical distancing, travel or movement restrictions, school/business closures and enforced quarantine to slow the spread of the disease and lessen its impact on the health system are often taken (WHO, 2018a). Thus, the negative psychological impact of a pandemic can be compounded by the public health measures introduced to contain the virus (Van Bortel et al. 2016; Holmes et al. 2020). Indeed, a series of recent reviews on the effects of quarantine and social isolation indicate they can lead to prolonged mental health difficulties (Brooks et al. 2020; Hossain et al. 2020; Loades et al. 2020).

Young people between 10 and 24 years of age account for almost a quarter of the total global population (Gupta, 2014; The World Bank, 2018). Adolescence and early adulthood are critical periods of development, which can shape the likelihood, severity and course of mental health problems (Kessler et al. 2007; Kessler et al. 2012). Many young people are attending school or university, which are among the first institutions to close as part of infection prevention measures, leaving them isolated from their peer groups as well as 
primary help-seeking and support facilities (Fegert \& Schuzle, in press; Stevenson et al. 2009; Van et al. 2010; WHO, 2017; Holmes et al. 2020; Kelly, 2020; WHO, 2017). Additionally, family distress is often high during a pandemic and young people may find themselves coping with feelings of distress and anxiety in the face of compromised support structures (Douglas et al. 2009).

On 11th March 2020, the WHO officially declared the spread of the coronavirus disease 2019 (COVID-19) as a pandemic. At the time of writing, there were over 4.3 million confirmed cases of COVID-19 across 188 countries/regions, with over 290000 associated deaths (John Hopkins University, 2020). Resulting public health responses have included widespread restrictions on social activity and closures of public spaces, schools and non-essential businesses (Bedford et al. 2020; Sohrabi et al. 2020). Emerging research on the COVID19 outbreak indicates that over half $(53.8 \%)$ of individuals rate the psychological impact of the pandemic as moderate to severe (Wang et al. 2020). Another nationwide study with 52730 respondents across 36 provinces in China, the country at the centre of the COVID-19 outbreak, found that over one-third (35\%) of individuals reported symptoms of psychological distress (Qiu et al. 2020). Others have suggested that individuals with confirmed and suspected cases of COVID-19 may experience fear of severe disease consequences and the contagion, and have increased risk of suicide (Li et al. 2020, Lin, 2020).

Exposure to the COVID-19 pandemic during a vulnerable developmental stage places young people at a greater risk of the negative psychological impacts of such an event (Holmes et al. 2020). The objective of this rapid review was to summarise the information available about the potential impact of a pandemic on the mental health of young people aged $12-25$ years. This age range was selected as it reflects international trends in current service provision for young people, research in this area (Hetrick et al. 2017) and the WHO definition of youth (United Nations [UN], 2013).

\section{Method}

\section{Rapid review methods}

A rapid review was conducted to capture relevant studies related to the research question. Rapid reviews condense the systematic review process to provide robust evidence-informed decisions in a cost-effective manner. This method is particularly appropriate when information and evidence is required quickly and in times of crisis (Tricco et al. 2017). The review was documented using the Preferred Reporting items for Systematic Reviews and Meta-Analysis
(PRISMA) guidelines. The protocol was registered with PROSPERO (CRD42020177796).

This review included all types of studies that explored how the mental health (outcome) of young people aged 12-25 years (population) could be affected by an exposure to a pandemic (exposure). This review was limited to studies relating to exposure to an infectious disease outbreak classified as either an 'epidemic' or 'pandemic', as these terms are often used interchangeably in the literature. This includes infectious disease outbreaks such as COVID-19, H1N1/swine flu, severe acute respiratory syndrome (SARS), Middle East respiratory syndrome (MERS), Ebola and HIV / AIDS. Studies examining treatments or risk factors for infectious diseases or exclusively focusing on populations such as healthcare workers were excluded. The focus was on studies where the majority of participants were aged 12-25 years, or where a sub-group of participants was clearly identified as being within this age range. For the purposes of the review, the WHO (2018b, para. 2) definition of mental health as 'a state of well-being in which every individual realises his or her own potential, can cope with the normal stresses of life, can work productively and fruitfully and is able to make a contribution to his or her own community'. There was no geographical restriction on papers. The search was restricted to English, peerreviewed abstracts and titles in PsycINFO (Proquest) and Medline (Proquest) from January 1985 to March 2020. Further details on the rapid review method and our search and selection strategy are provided in Appendix A.

\section{Consultation with experts}

In keeping with recommendations from the Cochrane Rapid Reviews Methods Group (Garritty et al. 2020), the research team sought input from $N=30$ youth mental health professionals working in a large youth mental health organisation based in Ireland in refining our research question. Respondents provided positive feedback to the research team and highlighted the potential application of the findings in the field.

\section{Data synthesis}

A quantitative synthesis proved to be inappropriate due to the heterogeneity of study designs, contexts and outcomes in the literature. Thus, a narrative synthesis across studies was used to identify key themes and concepts. Narrative synthesis refers to an approach to that relies chiefly on the use of words and text to summarise and explain the findings of the synthesis (Popay et al. 2006). First, the characteristics and findings of individual studies were tabulated, eligible studies were read and re-read independently by members of the 


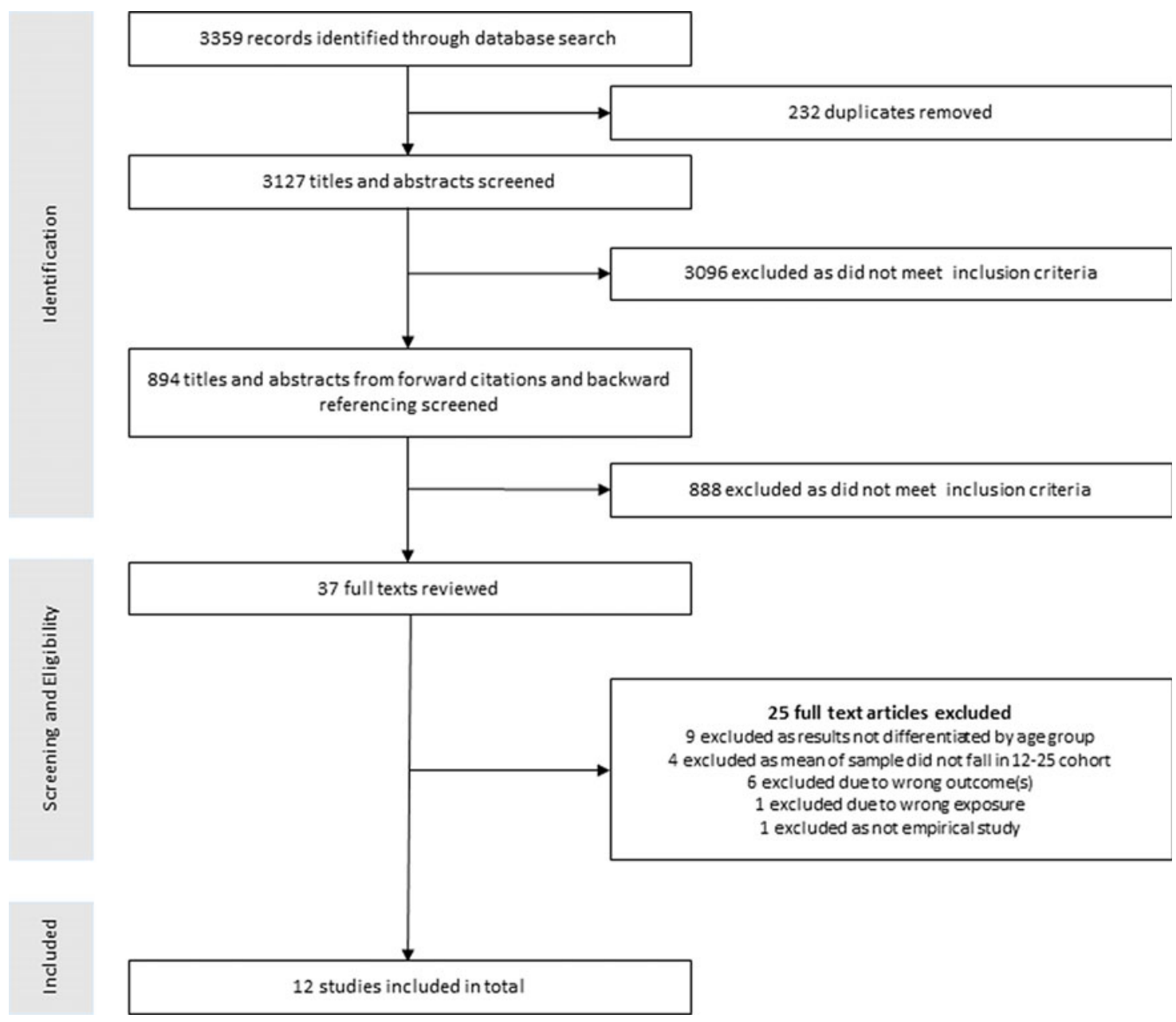

Fig. 1. Rapid review of peer-reviewed publications in the scientific literature: study selection.

research team and initial themes were generated (i.e. preliminary synthesis). As per Popay et al's. (2006) guidelines on narrative synthesis, variations in outcomes, study design, populations and content were noted, and relationships within and across studies were documented. Themes were then discussed and reviewed by the whole research group and against the full data set. As themes emerged from a review of the primary data, this remains an inductive approach (Atkins et al. 2008).

\section{Results}

\section{Search results}

Initial searches yielded 3,359 search results, which was reduced to 3,127 after duplicates were removed. The screening review process is illustrated in Fig. 1. Initially, two members of the research team (MT, $\mathrm{BMcK})$ reviewed the titles and abstracts of approximately half the papers each to make an initial assessment of relevance. Similar to Brown et al. (2020), following the initial search, papers that related to the HIV/AIDS pandemic were excluded as the mode of transmission is different (i.e. it is not an airborne transmission). A random sample of $10 \%$ of titles/ abstracts were examined by two additional reviewers (AB, AOR). Discrepancies $(N=17)$ were resolved through discussion. After this initial screening, 3,096 papers failed to meet the inclusion criteria, leaving 31 papers for full screening by two members of the research team (MT, BMcK). Forward and backward reference checking of key articles yielded a further six studies, leaving 37 papers for full screening.

After full screening, a further 25 papers failed to meet the inclusion criteria, leaving a final list of 12 papers for data extraction. Data were extracted onto a template by two researchers (MT, BMcK). Variables to be extracted comprised of the following: country of origin, study design, aims, method, participant characteristics, method of data analysis and key findings. This information was stored on a Microsoft Excel database. The remaining papers were examined using the appropriate Joanna Briggs critical appraisal checklist 
(Aromataris et al. 2015). These checklists have been widely used in rapid reviews and allow for the quick evaluation of study quality.

\section{Study characteristics and quality}

The studies included seven prevalence studies, three cross-sectional studies, one longitudinal study and one case-control study (see Tables 1 through 3). Most $(5 / 12)$ of the studies were undertaken in China, followed by Taiwan (2/12) and Hong Kong (2/12), with one each from Canada, Sweden and Saudi Arabia. Half of the studies (6/12) included 12-25-year-olds as part of larger studies with members of the public, $4 / 12$ were conducted with university students, one with medical students and one with children and young people who had developed narcolepsy after receiving the H1N1 vaccine. The majority of studies were related to the SARS outbreak (9/12), and one each to H1N1, MERS and COVID-19, respectively. Sample sizes were mostly modest, and varied from $N=38$ (Szakács et al. 2015) to $N=4,481$ (Leung et al. 2005). Two-thirds of the studies were rated as being of moderate quality $(8 / 12)$, while one-third were rated as high quality (4/12).

\section{Narrative synthesis}

Three major themes emerged from the narrative synthesis: prevalence of psychological difficulties among youth, factors moderating psychological difficulties and aspects of infectious disease outbreak causing distress.

\section{Prevalence of psychological difficulties}

There was some variation in findings regarding the prevalence of psychological difficulties among youth affected by an infectious disease outbreak. Four studies reported high anxiety or distress among young people recruited from the general population, university and health services during or following an outbreak (Bergeron \& Sanchez, 2005; Peng et al. 2010; Main et al. 2011; Szakács et al. 2015), while another found student status was predictive of greater psychological distress (Wang et al. 2020). However, other studies found older age groups reported higher levels of distress (25-44 -year-olds; Leung et al. 2005), perceived the pandemic had a greater impact on their mental health (25-34-year-olds and those aged 50+; Lau et al. 2005) or were more pessimistic (those aged 60+; Peng et al. 2005). Two studies found no age differences (Ko et al. 2006; Mihashi et al. 2009). Additionally, one study with university medicine students found that participants generally reported low levels of anxiety (Al-Rabiaah et al. 2020). A final study found young people in epidemic areas, which were described as 'the eye of the storm', were less anxious than those in non-epidemic areas, although the sample size was small (Xie et al. 2011). As shown in Tables 1 through 3 , there was significant variation in how mental health outcomes were measured, with some studies using author-designed measures, and others using standardised measures of anxiety [e.g. Depression Anxiety Stress Scale (DASS-21), Henry \& Crawford, 2005; State-Trait Anxiety Inventory (STAI), Spielberger et al. 1983; General Anxiety Disorder (GAD-7); Spitzer et al. 2007], psychological disorder [e.g. General Health Questionnaire (GHQ-30), Goldberg \& Williams, 1988] or distress [e.g. Brief Symptom Rating Scale (BSRS-5), Lung \& Lee, 2008; Impact of Event Scale (IES-R), Weiss, 2007].

\section{Factors moderating psychological difficulties}

Gender was only examined in three studies among the target age group. Two studies found female university students reported significantly higher levels of psychological distress than their male peers (Bergeron \& Sanchez, 2005; Al-Rabiaah et al. 2020). The results from a third study indicated male and female university students were equally affected by the SARS epidemic, although female students reported higher life satisfaction (Main et al. 2011). This study also found that, in general, all types of coping (i.e. active coping, avoidant coping and support focused coping) served as a buffer against the negative impact of stressors on perceived health, although female students reported less passive coping than their male peers. Additionally, Gan et al. (2004) reported that Chinese university students used less flexible coping strategies when dealing with SARS-related stress in comparison to daily life stresses, mirroring the coping reactions of individuals with depression.

It was notable that few studies asked participants to provide information on their physical health, given many individuals often experience physical illness during infectious disease outbreaks. Although four studies (Ko et al. 2006; Mihashi et al. 2009; Main et al. 2011; Wang et al. 2020) found self-reported health status was significantly associated with psychological difficulties, only one of these presented results for the target age group. Here, the authors observed a significant moderate positive correlation between psychological symptoms and general health among university students (Main et al. 2011). Another study looked at SARS-related vigilance among the general population, and found participants consistently thinking about whether or not they had contracted SARS was linked with higher levels of anxiety (Xie et al. 2011). Finally, two studies examined the relationship between the adoption of precautionary measures and psychological distress among young 
Table 1. Cross-sectional studies

\begin{tabular}{|c|c|c|c|c|c|c|c|c|}
\hline Citation & Sampling strategy & Participants & Region & Exposure & Aim and study design & Outcome measures & Main findings $12-25$ age group & $\begin{array}{l}\text { Joanna Briggs } \\
\text { quality appraisal }\end{array}$ \\
\hline $\begin{array}{l}\text { Mihashi } \\
\text { et al. } \\
\quad(2009)\end{array}$ & $\begin{array}{l}\text { Cluster sampling } \\
\text { from two } \\
\text { college } \\
\text { campuses; } \\
\text { Workers, } \\
\text { university } \\
\text { faculty and } \\
\text { family members } \\
\text { in campus 'A', } \\
\text { and students } \\
\text { located in } \\
\text { campus 'B' }\end{array}$ & $\begin{array}{l}N=187 \\
32 \% \text { female; } 54 \% \\
\text { male; }<1 \% \text { not } \\
\text { specified } \\
\text { Mean } \\
\quad 26.3 \pm 8 \text { years } \\
\text { Results } \\
\quad \text { disaggregated } \\
\text { by age: sub- } \\
\text { group } \\
\quad<23 \text { years }\end{array}$ & $\begin{array}{l}\text { Beijing, } \\
\text { China }\end{array}$ & SARS & $\begin{array}{l}\text { Investigated strategies for broad } \\
\text { mass isolation during outbreaks } \\
\text { of infectious diseases during the } \\
\text { SARS outbreak recovery period } \\
\text { Cross-sectional study using directly } \\
\text { delivered paper surveys }\end{array}$ & $\begin{array}{l}\text { Psychological disorders } \\
\text { (GHQ-30; Goldberg \& } \\
\text { Williams, 1988) }\end{array}$ & $\begin{array}{l}\text { No significant differences in } \\
\text { psychological disorders } \\
\text { between age groups } \\
(O R=.8) \\
\text { - } 24 \% \text { of those under } 23 \text { years } \\
\text { reported psychological } \\
\text { symptoms indicative of a } \\
\text { psychological disorder }\end{array}$ & Medium \\
\hline $\begin{array}{l}\text { Szakács } \\
\quad \text { et al. } \\
\quad(2015)\end{array}$ & $\begin{array}{l}\text { Purposive } \\
\text { sampling from } \\
\text { population- } \\
\text { based study }\end{array}$ & $\begin{array}{l}N=38 \\
55 \% \text { female; } 45 \% \\
\quad \text { male } \\
5-25 \text { years }\end{array}$ & Sweden & $\begin{array}{l}\text { H1N1 } \\
\quad \text { influenza }\end{array}$ & $\begin{array}{l}\text { Evaluated psychiatric comorbidity } \\
\text { and the cognitive profile of } \\
\text { children and adolescents with } \\
\text { narcolepsy in western Sweden } \\
\text { and the relationship of these } \\
\text { problems to H1N1 vaccination } \\
\text { Cross-sectional study using a test } \\
\text { battery of semi-structured } \\
\text { interviews }\end{array}$ & $\begin{array}{l}\text { Psychiatric comorbidity } \\
\text { [suite of measures to } \\
\text { assess meeting of ICD-10, } \\
\text { WHO, } 1993 \text { and DSM-IV } \\
\text { criteria; American } \\
\text { Psychiatric Association } \\
\text { (APA), 2000] } \\
\text { Cognitive Profile (Age- } \\
\text { appropriate Wechsler } \\
\text { Intelligence test battery) }\end{array}$ & $\begin{array}{l}\text { - Rates of psychiatric } \\
\text { difficulties were higher in } \\
\text { post-H1N1 vaccinated } \\
\text { participants than those with } \\
\text { non-post-H1N1 narcolepsy } \\
\left(O R=4.6^{\mathrm{a}}\right) \\
\text { - Major depression in post- } \\
\text { H1N1 vaccinated } \\
\text { participants was three times } \\
\text { higher than the prevalence in } \\
\text { the general adolescent } \\
\text { population }\end{array}$ & Medium \\
\hline $\begin{array}{r}\text { Xie } \text { et al. } \\
\quad(2011)\end{array}$ & $\begin{array}{l}\text { Cluster sampling } \\
\text { of residents in } \\
\text { infected and } \\
\text { non-infected } \\
\text { areas and } \\
\text { convenience } \\
\text { sampling }\end{array}$ & $\begin{array}{l}N=647 \\
35 \% \text { female; } 65 \% \\
\quad \text { male; }<1 \% \text { not } \\
\text { specified } \\
\text { Mean 23.8 years }\end{array}$ & China & SARS & $\begin{array}{l}\text { Examined effect of SARS crisis on } \\
\text { levels of distress during the } \\
\text { SARS epidemic in China } \\
\text { Cross-sectional study using online } \\
\text { surveys }\end{array}$ & $\begin{array}{l}\text { Anxiety (STAI-Form Y, } \\
\text { Chinese version; } \\
\text { Spielberger } \text { et al. 1983) } \\
\text { Imitative behaviour (author } \\
\text { designed measure) }\end{array}$ & $\begin{array}{l}\text { - Anxiety levels were higher } \\
\text { in non-infected areas than } \\
\text { quarantined or nearby } \\
\text { quarantined areas } \\
\text { - Anxiety was predicted } \\
\text { directly by SARS-related } \\
\text { vigilance, less willingness to } \\
\text { volunteer, perceived impact, } \\
\text { lower levels of perceived } \\
\text { knowledge and pessimism }\end{array}$ & Medium \\
\hline
\end{tabular}

SARS, severe acute respiratory syndrome; H1N1, influenza A sub-type H1N1; GHQ-30 , 30-item General Health Questionnaire; ICD-10, 10th revision of the International Statistical Classification of Diseases and Related Health Problems; DSM-IV, Diagnostic and Statistical Manual of Mental Disorders; STAI-Form Y, State-Trait Anxiety Inventory (Form Y); OR, odds ratio.

Joanna Briggs Quality appraisal rating is based on percentage of criteria met for appropriate study type, for the purposes of this study high $\geq 70 \%$, medium $=30-70 \%$ and low $\leq 30 \%$.

Effect size calculated from available data.

${ }^{b}$ Insufficient data available to calculate effect size. 
Table 2. Prevalence studies

\begin{tabular}{|c|c|c|c|c|c|c|c|c|}
\hline Citation & Sampling strategy & Participants & Region & Exposure & Aim and study design & Outcome measures & Main findings $12-25$ age cohort & $\begin{array}{l}\text { Joanna } \\
\text { Briggs } \\
\text { quality } \\
\text { appraisal }\end{array}$ \\
\hline $\begin{array}{l}\text { Al-Rabiaah et al. } \\
\quad(2020)\end{array}$ & $\begin{array}{l}\text { Random sampling } \\
\text { of university } \\
\text { medical students }\end{array}$ & $\begin{array}{l}N=174 \\
40 \% \text { female; } \\
\quad 60 \% \text { male } \\
\text { Mean } 21.6 \pm 1 \text { years }\end{array}$ & Saudi Arabia & MERS-CoV & $\begin{array}{l}\text { Examined the impact of MERS on } \\
\text { medical students' perception and } \\
\text { determinants of their psychological } \\
\text { distress during outbreak } \\
\text { Cross-sectional study using online } \\
\text { questionnaires }\end{array}$ & $\begin{array}{l}\text { Stress levels (1-10 } \\
\text { rating) } \\
\text { Anxiety levels } \\
\quad \text { (GAD-7; Spitzer } \\
\text { et al. 2007) }\end{array}$ & $\begin{array}{l}\text { - } 77 \% \text { reported minimal, } 18.4 \% \\
\text { reported mild and } 4.6 \% \text { reported } \\
\text { moderate levels of anxiety } \\
\text { Females had significantly higher } \\
\text { stress levels than males }\left(d=.4^{\mathrm{a}}\right) \\
\text { - Perceived social avoidance score } \\
(\beta=.2) \text {, improved hygiene habits } \\
(\beta=.3) \text { significantly predicted higher } \\
\text { stress levels } \\
\text { - Knowledge of MERS-CoV }(\beta=.1) \\
\text { agreeing with public fear }(\beta=.1) \text { and } \\
\text { number of resources accessed }(\beta=.1) \\
\text { did not significantly predict stress } \\
\text { levels }\end{array}$ & High \\
\hline $\begin{array}{l}\text { Bergeron, \& } \\
\quad \text { Sanchez (2005) }\end{array}$ & $\begin{array}{l}\text { Random sampling } \\
\text { of university } \\
\text { students }\end{array}$ & $\begin{array}{l}N=300 \\
73 \% \text { female } \\
18-23 \text { years } \\
\text { Mean } 21.1 \pm 5 \text { years }\end{array}$ & Canada & SARS & $\begin{array}{l}\text { Examined preferences and use of } \\
\text { various types of mass communication } \\
\text { media, anxiety levels of acquiring the } \\
\text { infection and general knowledge of } \\
\text { SARS in university students after the } \\
2003 \text { SARS outbreak } \\
\text { Cross-sectional study using online and } \\
\text { paper questionnaires }\end{array}$ & $\begin{array}{l}\text { Access and use of } \\
\text { mass } \\
\text { communication } \\
\text { media } \\
\text { Level of anxiety } \\
\text { (unspecified 7-item } \\
\text { scale) }\end{array}$ & $\begin{array}{l}\text { - } 43 \% \text { of student reported high levels } \\
\text { of anxiety } \\
\text { - Anxiety was not significantly } \\
\text { associated with the use intensity of any } \\
\text { type of mediab Gender (being female) } \\
\text { and area of residence (in the greater } \\
\text { Toronto area) was significantly } \\
\text { associated with high levels of anxiety }\end{array}$ & Medium \\
\hline Gan et al. (2004) & $\begin{array}{l}\text { Random sampling } \\
\text { of university } \\
\text { students }\end{array}$ & $\begin{array}{l}N=93 \\
58 \% \text { female; } \\
\quad 42 \% \text { male } \\
\text { Mean } 22.1 \pm 3 \text { years }\end{array}$ & $\begin{array}{l}\text { Beijing, } \\
\text { China }\end{array}$ & SARS & $\begin{array}{l}\text { Examined the coping flexibility of } \\
\text { university students in response to } \\
\text { SARS-related and daily life stressful } \\
\text { events } \\
\text { Cross-sectional study }\end{array}$ & $\begin{array}{l}\text { Coping flexibility } \\
\text { (CFQ; Cheng, 2001) } \\
\text { Daily life stressful } \\
\text { events (items from } \\
\text { ULES; Wang \& } \\
\text { Gan, 1994, ICSRLE; } \\
\text { Kohn et al. } 1990 \text { \& } \\
\text { SRRS; Reale, 1987) } \\
\text { SARS-related stressful } \\
\text { events (author } \\
\text { designed scale) }\end{array}$ & $\begin{array}{l}\text { Perceived controllability was } \\
\text { significantly lower for SARS-related } \\
\text { stress compared to daily life stress } \\
\left(d=1.2^{\mathrm{a}}\right) \\
\text { - No significant difference in perceived } \\
\text { effectiveness of coping behaviour } \\
\text { between SARS-related stress and daily } \\
\text { life stress }\left(d=.2^{\mathrm{a}}\right) \\
\text { - Coping flexibility was lower for } \\
\text { SARS-related stress than daily life } \\
\text { stress }^{\mathrm{b}}\end{array}$ & Medium \\
\hline
\end{tabular}


Table 2. (Continued)

\begin{tabular}{|c|c|c|c|c|c|c|c|c|}
\hline Citation & Sampling strategy & Participants & Region & Exposure & Aim and study design & Outcome measures & Main findings $12-25$ age cohort & $\begin{array}{l}\text { Joanna } \\
\text { Briggs } \\
\text { quality } \\
\text { appraisal }\end{array}$ \\
\hline Lau et al. (2005) & $\begin{array}{l}\text { Random sampling } \\
\text { of general } \\
\text { population in } \\
\text { Hong Kong } \\
\text { from phone } \\
\text { directories }\end{array}$ & $\begin{array}{l}\text { Survey } 1 \text { : } \\
N=863 \\
50 \% \text { female; } \\
\quad 50 \% \text { male } \\
18-60 \text { years } \\
\text { Survey } 2: \\
N=818 \\
50 \% \text { female; } \\
50 \% \text { male } \\
18-60 \text { years } \\
\text { Results } \\
\quad \text { disaggregated by } \\
\text { age: sub-group } \\
\text { 18-29 }\end{array}$ & $\begin{array}{l}\text { Hong Kong, } \\
\text { China }\end{array}$ & SARS & $\begin{array}{l}\text { Examined perceptions and mental } \\
\text { health effects of SARS on the general } \\
\text { population in Hong Kong during the } \\
\text { end phase of the epidemic } \\
\text { Cross-sectional study using two } \\
\text { telephone surveys }\end{array}$ & $\begin{array}{l}\text { Survey 1: } \\
\text { SARS-related } \\
\text { perceptions } \\
\text { Survey 2: } \\
\text { Psychological effects } \\
\quad \text { (self-reported) } \\
\text { Psychological effects } \\
\quad \text { (IES, Chinese } \\
\text { version; Horowitz } \\
\text { et al. 1979) } \\
\text { Mental health } \\
\quad(2 * \text { SF-36 } \\
\text { sub-scales; } \\
\text { Ware, 1992) }\end{array}$ & $\begin{array}{l}\text { - No significant difference in } \\
\text { psychological effects or quality of life } \\
\text { between age groups }{ }^{\mathrm{b}} \\
\text { - } 18-24 \text {-year-olds had significantly } \\
\text { lower odds of perceiving an overall } \\
\text { effect on mental health than those aged } \\
25-34\left(O R=.6^{\mathrm{a}}\right) \text { or } 50+\left(O R=.5^{\mathrm{a}}\right) \text {. } \\
\text { - No significant difference in having } \\
\text { trouble falling/staying asleep, having } \\
\text { a psychosomatic response or } \\
\text { perceived need for a psychiatrist or } \\
\text { psychologist between age groups }\end{array}$ & High \\
\hline Main et al. (2011) & $\begin{array}{l}\text { Cluster sampling } \\
\text { of university } \\
\text { students from } \\
\text { two public } \\
\text { universities. }\end{array}$ & $\begin{array}{l}N=381 \\
43 \% \text { male } \\
17-24 \text { years } \\
\text { Mean } 20.2 \pm 1 \text { years }\end{array}$ & $\begin{array}{l}\text { Beijing, } \\
\text { China }\end{array}$ & SARS & $\begin{array}{l}\text { Examined the main effects and } \\
\text { interactions between SARS-related } \\
\text { stressors and coping strategies and } \\
\text { Chinese college students' } \\
\text { psychological adjustment at the end } \\
\text { of the } 2003 \text { Beijing SARS epidemic } \\
\text { Cross-sectional study using paper } \\
\text { questionnaires }\end{array}$ & $\begin{array}{l}\text { Psychological } \\
\text { symptoms } \\
(4 * \text { SCL-90 } \\
\text { sub-scales, Chinese } \\
\text { version; Derogatis, } \\
\text { 1977) } \\
\text { Life satisfaction (Life } \\
\text { Satisfaction Scale; } \\
\text { Diener et al. 1985) } \\
\text { Perceived general } \\
\text { health (self-rated) }\end{array}$ & $\begin{array}{l}\text { - No significant gender difference in } \\
\text { psychological symptoms }\left(d=.01^{\mathrm{a}}\right) \text {. } \\
\text { Females reported significantly higher } \\
\text { levels of life satisfaction than males } \\
\left(d=.3^{\mathrm{a}}\right) \\
\text { - Experience of SARS-related stressors } \\
\text { was positively associated with } \\
\text { psychological symptoms }(\beta=.1) \\
\text { Interaction between SARS-related } \\
\text { stressors and coping predicted } \\
\text { perceived general health }(\beta=.1) \text { but } \\
\text { not psychological symptoms }(\beta=-.1) \\
\text { or life satisfaction }(\beta=-.02)\end{array}$ & Medium \\
\hline
\end{tabular}


Table 2. (Continued)

\begin{tabular}{|c|c|c|c|c|c|c|c|c|}
\hline Citation & Sampling strategy & Participants & Region & Exposure & Aim and study design & Outcome measures & Main findings $12-25$ age cohort & $\begin{array}{l}\text { Joanna } \\
\text { Briggs } \\
\text { quality } \\
\text { appraisal }\end{array}$ \\
\hline Peng et al. (2010) & $\begin{array}{l}\text { Stratified random } \\
\text { sampling of } \\
\text { general } \\
\text { population } \\
\text { based on } \\
\text { geographic area }\end{array}$ & $\begin{array}{l}N=1,278 \\
50 \% \text { female; } 50 \% \\
\quad \text { male } \\
18-89 \text { years } \\
\text { Mean } 41.6 \pm 17 \text { years } \\
\text { Results } \\
\quad \text { disaggregated by } \\
\quad \text { age: sub-group } \\
\quad 18-29\end{array}$ & Taiwan & SARS & $\begin{array}{l}\text { Explored post-crisis psychological } \\
\text { distress in Taiwan residents after the } \\
\text { SARS epidemic } \\
\text { Cross-sectional study using computer- } \\
\text { assisted telephone interview systems }\end{array}$ & $\begin{array}{l}\text { Change in perception } \\
\text { of life (pessimism) } \\
\text { Psychological distress } \\
\text { (BSRS-5; Lung \& } \\
\text { Lee, 2008) }\end{array}$ & $\begin{array}{l}\text { 18-29-year-olds had significantly } \\
\text { greater odds of having severe } \\
\text { psychological distress than those } \\
\text { aged } 50+\left(O R=2.0-2.5^{\mathrm{a}}\right) \\
\text { 18-29-year-olds had significantly } \\
\text { lower odds of feeling more } \\
\text { pessimistic after the resolution of the } \\
\text { SARS crisis than those aged } 60+ \\
\left(O R=.4^{\mathrm{a}}\right)\end{array}$ & High \\
\hline Wang et al. (2020) & $\begin{array}{l}\text { Snowball sampling } \\
\text { of general } \\
\text { population }\end{array}$ & $\begin{array}{l}N=1,210 \\
67 \% \text { female; } 33 \% \\
\quad \text { male } \\
12-59 \text { years } \\
\text { Results } \\
\quad \text { disaggregated by } \\
\text { age: sub-groups } \\
12-21 ; 21-31 \text { years }\end{array}$ & China & COVID-19 & $\begin{array}{l}\text { Examined levels of psychological } \\
\text { impact, anxiety, depression and stress } \\
\text { on the general public during the } \\
\text { initial stage of the COVID-19 } \\
\text { outbreak } \\
\text { Cross-sectional study using online } \\
\text { questionnaires }\end{array}$ & $\begin{array}{l}\text { Psychological impact } \\
\text { (IES-R; Weiss, } \\
\text { 2007) } \\
\text { Mental health status: } \\
\text { stress, anxiety and } \\
\text { depression (DASS- } \\
\text { 21; Henry \& } \\
\text { Crawford, 2005) }\end{array}$ & $\begin{array}{l}\text { - Student status was significantly } \\
\text { associated with higher psychological } \\
\text { impact }(\beta=.2) \text {, stress }(\beta=.1) \text {, anxiety } \\
(\beta=.2) \text { and depression }(\beta=.1) \\
\text { compared to those who were } \\
\text { employed } \\
\text { - No significant difference in } \\
\text { psychological impact, stress, anxiety } \\
\text { or depression between age groups } \\
(\beta=.1-.2)\end{array}$ & Medium \\
\hline
\end{tabular}

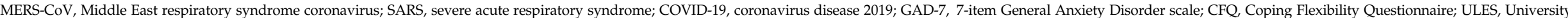

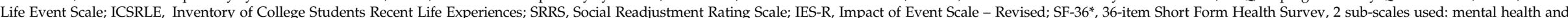
vitality /quality of life; SCL-90*, 90-item symptom checklist, 4 sub-scales used: somatisation, obsessive-compulsive, depressive and phobic/anxiety symptoms; BSRS-5, Brief Symptom Rating Scale; DASS-21, The Depression, Anxiety and Stress Scale; $d$, standardised mean difference; $\beta$, standardised regression coefficient; OR, odds ratio.

Joanna Briggs Quality appraisal rating is based on percentage of criteria met for appropriate study type, for the purposes of this study high $\geq 70 \%$, medium $=30-70 \%$, and low $\leq 30 \%$.

Effect size calculated from available data.

${ }^{\mathrm{b}}$ Insufficient data available to calculate effect size. 
Table 3. Other studies

\begin{tabular}{|c|c|c|c|c|c|c|c|c|}
\hline Citation & Sampling strategy & Participants & Region & Exposure & Aim and study design & Outcome measures & $\begin{array}{l}\text { Main findings } \\
12-25 \text { age cohort }\end{array}$ & $\begin{array}{l}\text { Joanna Briggs } \\
\text { quality appraisal }\end{array}$ \\
\hline $\begin{array}{l}\text { Leung } \\
\text { et al. } \\
\quad(2005)\end{array}$ & $\begin{array}{l}\text { Random sampling of } \\
\text { general population } \\
(18+)\end{array}$ & $\begin{array}{l}N=4,481 \\
\text { Gender: NR } \\
\text { 18-65+ years } \\
\text { Results disaggregated by } \\
\quad \text { age: } \\
\text { sub-group 18-24 years }\end{array}$ & $\begin{array}{l}\text { Hong } \\
\text { Kong, } \\
\text { China }\end{array}$ & SARS & $\begin{array}{l}\text { Examined psychological and } \\
\text { behavioural responses to the } \\
\text { SARS } \\
\text { outbreak over time } \\
\text { Longitudinal and repeated } \\
\text { cross-sectional design } \\
\text { involving } \\
6 \text { population based surveys } \\
\text { conducted using random digit } \\
\text { dialling }\end{array}$ & $\begin{array}{l}\text { Anxiety symptoms (STAI; } \\
\text { Spielberger et al. 1983) } \\
\text { Adoptions of } \\
\text { precautionary measures. }\end{array}$ & $\begin{array}{l}\text { Anxiety symptoms for } \\
\text { 18-24-year-olds } \\
\text { decreased over time } \\
\text { after infectious disease } \\
\text { outbreak }^{\text {b }} \\
\text { 18-24-year-olds had } \\
\text { lower anxiety } \\
\text { symptoms than } \\
\text { 25-44-year-olds at the } \\
\text { peak of the outbreak but } \\
\text { not at later time points }\end{array}$ & High \\
\hline $\begin{array}{l}\text { Ko et al. } \\
\quad(2006)\end{array}$ & $\begin{array}{l}\text { Random sampling of } \\
\text { general population } \\
(15+)\end{array}$ & $\begin{array}{l}N=1,473 \\
51 \% \text { female; } 49 \% \text { male } \\
15-50+\text { years } \\
\text { Results disaggregated by } \\
\text { age: sub-group } 15- \\
\quad 30 \text { years }\end{array}$ & Taiwan & SARS & $\begin{array}{l}\text { Examined the psychological } \\
\text { state of those impacted } v \text {. } \\
\text { those } \\
\text { not impacted by SARS } \\
\text { following its outbreak } \\
\text { Case control study using } \\
\text { telephone interviewing }\end{array}$ & $\begin{array}{l}\text { Depression level (TDQ; } \\
\text { Lee et al. 2020) } \\
\text { Self-Perceived Health } \\
\text { Questionnaire } \\
\text { Neighbourhood } \\
\text { Relationship } \\
\text { Questionnaire }\end{array}$ & $\begin{array}{l}\text { No significant } \\
\text { difference in depression } \\
\text { level between age } \\
\text { groups in those } \\
\text { impacted by SARS } \\
\left(d<.01^{\mathrm{a}}\right)\end{array}$ & Medium \\
\hline
\end{tabular}

SARS, severe acute respiratory syndrome; STAI, State-Trait Anxiety Inventory; TDQ, Taiwanese Depression Questionnaire; $d$, standardised mean difference.

Joanna Briggs Quality appraisal rating is based on percentage of criteria met for appropriate study type, for the purposes of this study high $\geq 70 \%$, medium $=30-70 \%$ and low $\leq 30 \%$.

Effect size calculated from available data.

Insufficient data available to calculate effect size. 
people. Results from both studies indicated that the adoption of precautionary measures such as avoiding others and greater change in hygiene habits was significantly associated with higher levels of anxiety/stress (Xie et al. 2011; Al-Rabiaah et al. 2020).

\section{Aspects of infectious disease outbreak causing distress}

The timing of data collection varied across studies. With the exception of one longitudinal study, which comprised multiple phases of data collection with members of the general population during and after a SARS outbreak (Leung et al. 2005), most studies were conducted when an outbreak had been controlled or after the resolution of this event. While the longitudinal study did show an overall decrease in anxiety in a population from the peak of an epidemic to post-epidemic, and this trend was observed among 18-24-year-olds, results were not significant for this age group (Leung et al. 2005)

In addition to studies looking at general difficulties during a pandemic, others focused on events linked to an infectious disease outbreak. A small number focused on social isolation or quarantine among youth. As noted earlier, in one study, the authors found that participants in epidemic areas were generally less anxious than those in non-epidemic areas (Xie et al. 2011). A second study found exposure to more SARS stressors, including having to cancel planned activities, predicted psychological difficulties among university students (Main et al. 2011). Other studies pointed to a relationship between being quarantined or living with restrictions and psychological well-being, but results were not disaggregated by age (Ko et al. 2006; Mihashi et al. 2009; Peng et al. 2010).

Finally, there were two studies that looked at specific factors associated with a pandemic. One study examined media use and its link to mental health among university students, but found anxiety levels were not associated with the use intensity of any type of media (Bergeron \& Sanchez, 2005). Another study examined psychological difficulties among children and adolescents who had developed narcolepsy after receiving a vaccine for $\mathrm{H} 1 \mathrm{~N} 1$, and found higher prevalence of psychiatric disorders among this group compared to those who had developed narcolepsy due to other reasons (Szakács et al. 2015).

\section{Discussion}

The purpose of this rapid review was to synthesise and describe the available evidence on the potential impact of a pandemic on young people's mental health. There is generally consensus in the literature that rates of anxiety and depression across countries among adolescents and young adults have increased (Collishaw, 2015;
Mojtabai et al. 2016; Dooley et al. 2019; Patalay \& Gage, 2019). How young people's mental health is affected by an infectious disease outbreak and the public health measures to control such an outbreak is unclear. This review revealed some studies in this area point to heightened vulnerability among youth, others suggest adults are more affected - possibly due to increased physical health risks (Mackay \& Arden, 2015) - and a small number report no age differences. The research almost consistently indicates females report higher levels of distress, which mirrors the broader literature with this age group (Dooley et al. 2019; Patalay \& Gage, 2019; Wiens et al. 2020).

The disparity observed may be somewhat explained by the different instruments used to assess mental health/psychological well-being. While several studies used standardised questionnaires to examine a particular aspect of distress, others used one-item authordesigned measures. It is also worth noting that most $(75 \%)$ of the studies were conducted in Chinese or other Eastern cultures, where a number of recent infectious disease outbreaks have occurred. Eastern and western cultures typically respond differently to negative emotions (Furlong \& Finnie, in press). Individuals from collective cultures tend to report more somatic symptoms than psychological symptoms (Ryder et al. 2008), and coping strategies are also likely to vary depending on culture (Chun et al. 2006), pointing to the need to consider the larger social and cultural context in addition to the situational context of a pandemic (Wong et al. 2006). Indeed, two studies point to the importance of adaptive coping styles in responding to adversity during an infectious disease outbreak. Maladaptive coping is a risk factor for the development of psychological difficulties after a pandemic or natural disaster (Coetzee \& Spangenberg, 2003; Naushad et al. 2019).

It was surprising that the majority of the research included in this review was conducted in the latter stages or after an infectious disease outbreak. None of the studies reviewed included data collection points prior to and after an infectious disease outbreak, meaning the ability to infer changes in youth mental health as a direct result of the outbreak is significantly limited. There is a real need to conduct more longitudinal research, particularly prior to and during the peak stages of an infectious disease outbreak, when young people are most likely to be affected by public health measures or feel particularly anxious about their physical health. Although previous research has established a link between the impact of social isolation, quarantine and restricted movements and distress (Brooks et al. 2020; Hossain et al. 2020; Loades et al. 2020), we could not draw firm conclusions from this review on how young people are affected by such measures during a pandemic. The COVID-19 pandemic is much more 
widespread than the other infectious disease outbreaks described in many of the papers included in this review, and the long-term economic effects are likely to be more significant, particularly for young people [Oswald \& Powdthavee, 2020; Institute for Fiscal Studies (ISF), 2020]. Previous research has indicated youth and parent unemployment can have a significant psychological impact on young people (Fergusson et al. 2001; Virtanen et al. 2016). Conversely, the successful recovery of national economies appears to crucially depend on the mental health of the population (WHO, 2011).

It is worth noting that most of the studies included in this review used convenience, non-representative samples. Although seven studies reported some element of random sampling, the samples were typically restricted to a particular geographic or educational setting. Only two studies reported random sampling based on a specified sampling frame, thus limiting the ability to make accurate inferences about prevalence. The studies were also typically comprised of university students, and none were conducted solely with 12-25-year-olds. Only four studies included young people under the age of 18 , meaning we are limited in our ability to make inferences about prevalence particularly in terms of how adolescents may be affected by a pandemic. Additional research with young people with preexisting mental health difficulties or those experiencing challenges with regards to their personal, family or social circumstances are warranted, as this group may be disproportionally affected by the mediumand long-term social effects of COVID-19, and resource allocation for youth mental health services is generally insufficient (Brown et al. 2020; Furlong \& Finnie, in press; Li et al. 2020). The voice of young people is also notably absent from the literature on this topic. Patient and public involvement is critical to understanding people's lived experiences, yet the methods adopted in the existing body of research do not actively promote youth voice. It is important any research with young people is ethically robust and researchers view COVID-19 mental health research as a sensitive topic, where attention is paid to the safeguards needed to protect the well-being of participants (Townsend et al. 2020). This is particularly salient for research with young people under the age of 18, where legal and developmental considerations limit their capacity to consent independently and parental support may be required (Hiriscau et al. 2016).

\section{Strengths/limitations of study}

This review is the first to focus on the mental health impacts of a pandemic on the 12-25-year-old cohort, which is a target age group for a growing number of youth mental health services internationally (Hetrick et al. 2017). Incorporating a consultation with mental health professionals to refine the research question, collaborating with a young person as an author on the rapid review team, adopting a systematic process of study selection and rigorous synthesis methods are all key strengths of the review.

However, the review conclusions are ultimately limited by the quality of the primary studies reviewed. Although all of the studies identified in this review were rated as moderate or high in terms of quality, convenience sampling, an absence of strategies to deal with confounding factors, variation in measures used to assess the primary outcome (mental health) and heterogeneity of outcome measures in the studies identified are all limitations of the review. As noted above, there is also a notable absence of studies with adolescents or incorporating youth perspectives in the reviewed studies. In addition, the predominance of crosssectional data gathered in Eastern cultures in the period before/after a pandemic limits our ability to draw conclusions about the immediate or subsequent long-term impacts of a pandemic on youth mental health. Further, slightly more than half of the studies included respondents outside the 12-25-year-old age group, most of which contained only limited, albeit valuable, information that was disaggregated for this age cohort. Additionally, in order to quickly collate the evidence available, this review employed single-reviewer screening with $10 \%$ verification by a second reviewer, which is common in rapid reviews (Abou-Setta et al. 2016). Finally, the review focused on peer-reviewed, English language publications and may not have identified all related existing and emerging published/ unpublished publications related to pandemics.

\section{Recommendations for practice}

Few studies have considered the collective impact of biological, social and psychological risk and protective factors on youth mental health, meaning our ability to make recommendations about how to effectively intervene and impact on young people's mental health during a pandemic is limited. However, some considerations for practice and policy are evident. First, this review highlights mental health should be considered as part of a holistic response to the COVID-19 outbreak. Second, while cultural context must be considered, there are indicators that adaptive coping styles can support young people's capacity to navigate through an uncontrollable event such as a pandemic, pointing to an area of intervention for mental health service providers. Psychological interventions incorporating cognitive behavioural therapy or problem-solving therapy may be valuable, and could be delivered online. Delivery of online services and the integration 
of e-therapy tools have begun in many countries as a result of the COVID-19 outbreak (Wind et al. 2020). Community-based workshops or health promotion campaigns could also focus on the promotion of adaptive coping styles. Finally, this review highlights the need to take factors such as age and gender into account when delivering mental health campaigns to support populations in the aftermath of COVID-19.

\section{Conclusion}

During an infectious disease outbreak, the focus of research and action is often on the medical and public health communities, where it has typically (rightly) been on the identification of the responsible agent, clinical presentation and treatment of the disease (Leung et al. 2005). However, it is important to pay attention to the ways a pandemic can impact on mental health. To the best of the author's knowledge, this is the first time the evidence on young people's mental health during a pandemic has been synthesised. On the basis of the review, we are unable to determine the extent by which - if at all - young people's mental health is affected by a pandemic, what factors may mitigate the impact of a pandemic on mental health, and how culture/context could affect this impact. The review highlights there has been minimal consideration of how this group can been affected by a pandemic, and points to an urgent need for more research on this area, particularly with adolescents. The COVID-19 crisis has been described as 'unprecedented, prolonged and unpredictable' (Pūras, 2020) and the impact on youth well-being needs to be considered as a priority.

\section{Conflict of interest}

The authors of this paper were, at the time of writing, employed or working with Jigsaw - The National Centre for Youth Mental Health, whose mission is to advance the mental health of young people in Ireland (aged 12-25) by influencing change, strengthening communities and delivering services through an evidence-informed and early intervention approach.

\section{Ethical standards}

The authors assert that all procedures contributing to this work comply with the ethical standards of the relevant national and institutional committee on human experimentation with the Helsinki Declaration of 1975, as revised in 2008.

\section{Financial support}

The work of the authors was supported by government funding provided by the Irish Health Service Executive (HSE).

\section{Author contributions}

All authors contributed to the design and implementation of the research, to the analysis of the results and to the writing of the manuscript. One of the authors of this manuscript was a young person, in the age category under investigation in this review.

\section{References}

Abou-Setta AM, Jeyaraman MM, Attia A, Al-Inany HG, Ferri M, Ansari MT, Garritty CM, Bond K, Norris SL (2017). Methods for developing evidence reviews in short periods of time: a scoping review. PLOS ONE 12, e0172372.

Al-Rabiaah A, Teemsah MH, Al-Eyadhy AA, Hasan GM, Al-Zamil F, Al-Subaie S Alsohime F, Jamal A, ALhaboob A, Al-Saadi B, Somily AM (2020). Middle East Respiratory Syndrome-Corona Virus (MERS-CoV) associated stress among medical students at a university teaching hospital in Saudi Arabia. Journal of Infection and Public Health 13, 687-691.

Aromataris E, Fernandez R, Godfrey CM, Holly C, Khalil H, Tungpunkom P (2015). Summarizing systematic reviews: methodological development, conduct and reporting of an umbrella review approach. International Journal of Evidence Based Healthcare 13, 132-140.

Atkins S, Lewin S, Smith H, Engel M, Fretheim A, Volmink J (2008). Conducting a meta-ethnography of qualitative literature: lessons learnt. BMC Medical Research Methodology 8(1), 21.

Bedford J, Enria D, Giesecke J, Heymann DL, Ihekweazu C, Kobinger G, Lane HC, Memish Z, Oh MD, Sall AA, Schuchat A, Ungchusak K, Wieler LH (2020). COVID-19: towards controlling of a pandemic. The Lancet 395, 1015-1018.

Bergeron SL, Sanchez AL (2005). Media effects on students during SARS outbreak. Emerging Infectious Diseases 11, 732-734.

Brooks SK, Webster RK, Smith LE, Woodland L, Wessely S, Greenberg N, Rubin GJ (2020). The psychological impact of quarantine and how to reduce it: rapid review of the evidence. The Lancet 395, 912-920.

Brown E, Gray R, Lo Monaco S, O'Donoghue B, Nelson B, Thomspon A, Francey S, McGorry P (2020). The potential impact of COVID-19 on psychosis: a rapid review of contemporary epidemic and pandemic research. Schizophrenia Research. S0920-9964(20)30257-7.

Cheng C (2001). Assessing coping flexibility in real-life and laboratory settings: a multimethod approach. Journal of personality and social psychology 80, 814.

Chun CA, Moos RH, Cronkite RC (2006). Culture: a fundamental context for the stress and coping paradigm. In Handbook of Multicultural Perspectives on Stress and Coping (ed. P. T. P Wong, L. C. J. Wong), pp. 29-53. Springer: US. 
Coetzee M, Spangenberg J (2003). Coping styles and quality of life in people with HIV/AIDS: a review. Acta Academica 35, 205-222.

Collishaw S (2015). Annual research review: secular trends in child and adolescent mental health. Journal of Child Psycholy \& Psychiatry 56, 370-93.

Derogatis LR (1977). SCL-90-R Administration, Scoring and Procedures Manual. Clinical Psychometric Research: Baltimore, MD.

Dooley B, O'Connor C, Fitzgerald A, O' Reilly A (2019). My World Survey 2: The National Study of Youth Mental Health. UCD School of Psychology \& Jigsaw: Dublin, Ireland.

Doshi P (2011). The elusive definition of pandemic influenza. Bulletin of the World Health Organization 89, 532-538.

Douglas PK, Douglas DB, Harrigan DC, Douglas KM (2009). Preparing for pandemic influenza and its aftermath: mental health issues considered. International Journal of Emergency Mental Health 11, 137.

Fegert JM, Schulze U (2020). Covid-19 and its impact on child and adolescent psychiatry - a German and personal perspective. Irish Journal of Psychological Medicine, 1-3. doi: 10.1017/ipm.2020.43.

Fergusson DM, Horwood LJ, Woodward LJ (2001). Unemployment and psychosocial adjustment in young adults: causation or selection? Social Science \& Medicine 53, 305-320.

Furlong Y, Finnie T. Culture counts: the diverse effects of culture and society on mental health amidst COVID-19 outbreak in Australia. Irish Journal of Psychological Medicine.

Gan Y, Liu Y, Zhang Y (2004). Flexible coping responses to severe acute respiratory syndrome-related and daily life stressful events. Asian Journal of Social Psychology 7, 55-66.

Garritty C, Gartlehner G, Kamel C, King V, Nussbaumer-Streit B, Stevens A, Hamel C, Affengruber L (2020). Interim Guidance from the Cochrane Rapid Reviews Methods Group. March 2020. (https://methods.cochrane.org/rapidreviews/sites/ methods.cochrane.org.rapidreviews/files/public/ uploads/cochrane_rr_-_guidance-23mar2020-final.pdf).

Goldberg DP, Williams P (1988). User's guide to the General Health Questionnaire. NFER-Nelson: Windsor.

Gupta MD (2014). The power of 1.8 Billion: Adolescents, Youth and the Transformation of the Future. United Nations Population Fund: New York.

Guze SB (1995). Diagnostic and Statistical Manual of Mental Disorders, 4th ed. (DSM-IV). American Journal of Psychiatry 152, 1228-1228.

Hall RC, Hall RC, Chapman ML (2008). The 1995 Kikwit Ebola outbreak: lessons hospitals and physicians can apply to future viral epidemics. General Hospital Psychiatry 30, 446-452.

Henry JD, Crawford JR (2005). The short-form version of the Depression Anxiety Stress Scales (DASS-21): construct validity and normative data in a large non-clinical sample. British Journal of Clinical Psychology 44, 227-239.
Hetrick SE, Bailey AP, Smith KE, Malla A, Mathias S, Singh SP, O'Reilly A, Verma SK, Benoit L, Fleming TM, Moro MR, Rickwood D, Duffy J, Eriksen T, Illback R, Fisher CA, McGorry PD (2017). Integrated (one-stop shop) youth health care: best available evidence and future directions. Medical Journal of Australia 207, S5-S18.

Hiriscau EI, Stingelin-Giles N, Wasserman D, Reiter-Theil S (2016). Identifying ethical issues in mental health research with minors adolescents: results of a Delphi study. International Journal of Environmental Research and Public Health 13, 489.

Holmes EA, OConnor R, Perry VH, Tracey I, Wessely S, Arseneault L (2020). Multidisciplinary research priorities for the COVID-19 pandemic: A call for action for mental health science. The Lancet Psychiatry 7: 547-560.

Horowitz M, Wilner N, Alvarez W (1979). Impact of event scale: a measure of subjective stress. Psychosomatic Medicine 41, 209-218.

Hossain MM, Sultana A, Purohit N (2020). Mental health outcomes of quarantine and isolation for infection prevention: a systematic umbrella review of the global evidence. (https: / / ssrn.com/abstract $=3561265$ ) or doi: 10.2139/ssrn.3561265. Accessed 15 May 2020.

Institute for Fiscal Studies [ISF] (2020). Sector shutdowns during the coronavirus crisis: which workers are most exposed?. (https://www.ifs.org.uk/publications/14791). Accessed 15 May 2020.

John Hopkins University (2020). COVID-19 Map. Johns Hopkins Coronavirus Resource Center. (https://coronavirus. jhu.edu/map.html). Accessed 15 May 2020.

Kelly BD (2020). Plagues, pandemics and epidemics in Irish history prior to COVID-19 (coronavirus): what can we learn? Irish Journal of Psychological Medicine, 1-6. doi: 10. 1017/ipm.2020.25.

Kelly H (2011). The classical definition of a pandemic is not elusive. Bulletin of the World Health Organization 89, 540-541.

Kessler RC, Angermeyer M, Anthony JC, De Graaf R, Demyttenaere K, Gasquet L, De Girolamo G, Gluzman S, Gureje O, Haro JM (2007). Lifetime prevalence and age-of-onset distributions of mental disorders in the World Health Organization's World Mental Health Survey Initiative. World Psychiatry 6, 168.

Kessler RC, Avenevoli S, McLaughlin KA, Green JG, Lakoma MD, Petukhova M, Pine D, Sampson NA, Zaslavsky AM, Merikangas, KR (2012). Lifetime co-morbidity of DSM-IV disorders in the US National Comorbidity Survey Replication Adolescent Supplement (NCS-A). Psychological Medicine 42, 1-14.

Ko CH, Yen CF, Yen JY, Yang MJ (2006). Psychosocial impact among the public of the severe acute respiratory syndrome epidemic in Taiwan. Psychiatry and Clinical Neurosciences 60, 397-403.

Kohn PM, Lafreniere K, Gurevich M (1990). The inventory of college students' recent life experiences: A decontaminated hassles scale for a special population. Journal of Behavioral Medicine 13, 619-630. 
Lau JTF, Yang X, Pang E, Tsui HY, Wong E, Yun KW (2005). SARS-related perceptions in Hong Kong. Emerging Infectious Diseases 11, 417-424.

Lee Y, Yang MJ, Lai TJ, Chiu NM, Chau T (2000). Development of the Taiwanese depression questionnaire. Chang Gung Medical Journal 23, 688-694.

Leung GM, Ho LM, Chan SK, Ho SY, Bacon-Shone J, Choy RY, Hedley AJ, Lam TH, Fielding R (2005). Longitudinal assessment of community psychobehavioral responses during and after the 2003 outbreak of severe acute respiratory syndrome in Hong Kong. Clinical Infectious Diseases 40, 1713-1720.

Li W, Yang Y, Liu ZH, Zhao YJ, Zhang QG, Zhang L, Cheung T, Xiang YT (2020). Progression of mental health services during the COVID-19 outbreak in China. International Journal of Biological Sciences 16, 1732-1738.

Lin CY (2020). Social reaction toward the 2019 novel coronavirus (COVID-19). Social Health and Behavior 3, 1.

Loades, ME, Chatburn E, Higson-Sweeney N, Reynolds S, Shafran R, Brigden A, Linney C, McManus M, Borwick C, Crawley E (2020). Rapid review: the impact of social isolation and loneliness on the mental health of children and adolescents in the context of COVID-19. (https://osf.io/p8q3d). Accessed 15 May 2020.

Lung FW, Lee MB (2008). The five-item brief-symptom rating scale as a suicide ideation screening instrument for psychiatric inpatients and community residents. BMC Psychiatry 8, 53-53.

Mackay IM, Arden KE (2015). MERS coronavirus: diagnostics, epidemiology and transmission. Viroglogy Journal 12, 222.

Madhav N, Oppenheim B, Gallivan M, Mulembakani P, Rubin E, Wolfe N (2017). Pandemics: risks, impacts, and mitigation. In Disease Control Priorities: Improving Health and Reducing Poverty. (eds. D. T. Jamison, H. Gelband, S. Horton, P. Jha, R. Laxminarayan, C. N. Mock, $\&$ R. Nugent). The International Bank for Reconstruction and Development/The World Bank: Washington, DC.

Main A, Zhou Q, Ma Y, Luecken LJ, Liu X (2011). Relations of SARS-related stressors and coping to Chinese college students' psychological adjustment during the 2003 Bbeijing SARS epidemic. Journal of Counseling Psychology 58, 410-423.

Mihashi M, Otsubo Y, Yinjuan X, Nagatomi K, Hoshiko M, Ishitake T (2009). Predictive factors of psychological disorder development during recovery following SARS outbreak. Health Psychology 28, 91-100.

Mojtabai R, Olfson M, Han B (2016). National trends in the prevalence and treatment of depression in adolescents and young adults. Pediatrics 138, e20161878-e20161878.

Naushad VA, Bierens JJ, Nishan KP, Firjeeth CP, Mohammad OH, Maliyakkal AM, Chalihadan S, Schreiber MD (2019). A systematic review of the impact of disaster on the mental health of medical responders. Prehospital and Disaster Medicine 34, 632-643.

Oswald AJ, Powdthavee N (2020). The case for releasing the young from lockdown: A briefing paper for policymakers. IZA Discussion Paper No. 13113. (https://ssrn.com/ abstract=3573283). Accessed 15 May 2020.
Patalay P, Gage SH (2019). Changes in millennial adolescent mental health and health-related behaviours over 10 years: a population cohort comparison study. International Journal of Epidemiology 48, 1650-1664.

Peng EYC, Lee MB, Tsai ST, Yang CC, Morisky DE, Tsai LT, Weng YL, Lyu SY (2010). Population-based post-crisis psychological distress: an example from the SARS outbreak in Taiwan. Journal of the Formosan Medical Association 109, 524-532.

Popay J, Roberts H, Sowden A, Petticrew M, Arai, L, Rodgers M, Britten N, Roen K, Duffy S (2006). Guidance on the conduct of narrative synthesis in systematic reviews. A Product from the ESRC Methods Programme Version 1, b92.

Puras D (2020). COVID-19 and mental health: challenges ahead demand changes. Health and Human Rights Journal. (https:/ / www.hhrjournal.org/2020/05/covid-19and-mental-health-challenges-ahead-demand-changes/). Accessed 13 July 2020.

Qiu J, Shen B, Zhao M, Wang Z, Xie B, Xu Y (2020). A nationwide survey of psychological distress among Chinese people in the COVID-19 epidemic: implications and policy recommendations. General Psychiatry 33, e100213.

Reale J (1987). Life changes: can they cause disease? Nursing $17,52$.

Rubin GJ, Potts HWW, Michie S (2010). The impact of communications about swine flu (influenza A H1N1v) on public responses to the outbreak: results from 36 national telephone surveys in the UK. Health Technology Assessment 14, 183-266.

Ryder AG, Yang J, Zhu X, Yao S, Yi J, Heine SJ, Bagby RM (2008). The cultural shaping of depression: somatic symptoms in China, psychological symptoms in North America?. Journal of Abnormal Psychology 117, 300-313.

Sim K, Huak Chan Y, Chong PN, Chua HC, Wen Soon S (2010). Psychosocial and coping responses within the community health care setting towards a national outbreak of an infectious disease. Journal of Psychosomatic Research 68, 195-202.

Sohrabi C, Alsafi Z, O'Neill N, Khan M, Kerwan A, Al-Jabir A, Iosifidis C, Agha R (2020). World health organization declares global emergency: a review of the 2019 novel coronavirus (COVID-19). International Journal of Surgery 76, 71-76.

Spielberger CD, Gorsuch RL (1983). State-Trait Anxiety Inventory for Adults: Manual and Sample: Manual, Instrument and Scoring Guide. Consulting Psychologists Press: Palo Alto, CA.

Spitzer RL, Kroenke K, Williams JB, Löwe B (2006). A brief measure for assessing generalized anxiety disorder: the GAD-7. Archives of Internal Medicine 166, 1092-1097.

Stevenson E, Barrios L, Cordell R, Delozier D, Gorman S, Koenig LJ, Odom E, Polder J, Randolph J, Shimabukuro T, Singleton C (2009). Pandemic influenza planning: addressing the needs of children. American Journal of Public Health 99, S255-S260. 
Szakács A, Hallböök T, Tideman P, Darin N, Wentz E (2015). Psychiatric comorbidity and cognitive profile in children with narcolepsy with or without association to the H1N1 Influenza vaccination. Sleep 38, 615-621.

The World Bank (2018). Health Nutrition and Population Statistics | DataBank. (https://databank.worldbank.org/ source/health-nutrition-and-population-statistics). Accessed 15 May 2020.

Townsend E, Nielsen E, Allister R, Cassidy SA (2020). Key ethical questions for research during the COVID-19 pandemic. The Lancet Psychiatry 7, 381-383.

Tricco AC, Langlois EV, Straus SE (2017). Rapid Reviews to Strengthen Health Policy and Systems: A Practical Guide. (https://www.who.int/alliance-hpsr/resources/ publications/alliancehpsr_rrguide_trainingslides.pdf). Accessed 15 May 2020.

United Nations [UN] (2013). Definition of Youth [fact sheet]. (https://www.un.org/esa/socdev/documents/youth/ fact-sheets/youth-definition.pdf). Accessed 15 May 2020.

Van Bortel T, Basnayake A, Wurie F, Jambai M, Koroma AS, Muana AT, Hann K, Eaton J, Martin S, Nellums LB (2016). Psychosocial effects of an Ebola outbreak at individual, community and international levels. Bulletin of the World Health Organization 94, 210-214.

Van D, McLaws ML, Crimmins J, MacIntyre CR, Seale H (2010). University life and pandemic influenza: attitudes and intended behaviour of staff and students towards pandemic (H1N1) 2009. BMC Public Health 10, 130.

Virtanen P, Hammarström A, Janlert U (2016). Children of boom and recession and the scars to the mental health-a comparative study on the long-term effects of youth unemployment. International Journal for Equity in Health 15, 14.

Wang D, Gan Y (1994). Cognitive-knowledge system model of perceived controllability on life events. Acta Psychologica Sinica 26, 69-76.

Wang C, Pan R, Wan X, Tan Y, Xu L, Ho CS, Ho RC (2020). Immediate psychological responses and associated factors during the initial stage of the 2019 Coronavirus Disease (COVID-19) epidemic among the general population in China. International Journal of Environmental Research and Public Health 17, 1729.

Ware JE, Sherbourne CD (1992). The MOS 36-ltem short-form health survey (SF-36). Medical Care 30, 473-483.

Weiss DS (2007). The impact of event scale: revised. In Cross-Cultural Assessment of Psychological Trauma and PTSD, pp. 219-238. Springer. doi: 10.1007/978-0387-70990-1_10.

Wiens K, Bhattarai A, Pedram P, Dores A, Williams J, Bulloch A, Patten S (2020). A growing need for youth mental health services in Canada: examining trends in youth mental health from 2011 to 2018. Epidemiology and Psychiatric Sciences 29, E115.

Wind TR., Rijkeboer M, Andersson G, Riper H (2020). The COVID-19 pandemic: the 'black swan' for mental health care and a turning point for e-health. Internet Interventions 20, 100317.
Wong PTP, Wong LCJ, Scott C (2006). Beyond stress and coping: the positive psychology of transformation. In Handbook of Multicultural Perspectives on Stress and Coping; Handbook of Multicultural Perspectives on Stress and Coping (ed. P. T. P. Wong, L. C. J. Wong), pp. 1-26. Wong-Spring Publications: Washington, DC.

World Health Organization [WHO] (1993). The ICD-10 Classification of Mental and Behavioural Disorders: Diagnostic Criteria for Research (Vol. 2). World Health Organization: Geneva.

World Health Organization [WHO] (2011). Impact of Economic Crises on Mental Health. (http:/ / www.euro.who. int/_data/assets/pdf_file/0008/134999/e94837.pdf? ua=1). Accessed 15 May 2020.

World Health Organization [WHO] (2017). Pandemic Influenza Risk Management: A WHO Guide to Inform and Harmonize National and International Pandemic Preparedness and Response. (http://www.who.int/influenza/ preparedness/pandemic/influenza_risk_management_ update2017/en/). Accessed 15 May 2020.

World Health Organization [WHO] (2018a). A Checklist for Pandemic Influenza Risk and Impact Management: Building Capacity for Pandemic Response. (http:/ / www.who.int/ influenza/preparedness/pandemic/ influenza_risk_management_checklist_2018/en/). Accessed 15 May 2020.

World Health Organization [WHO] (2018b). Mental Health: Strengthening Our Response. (https:/ / www.who.int/newsroom/fact-sheets/detail/mental-health-strengthening-ourresponse). Accessed 15 May 2020.

World Health Organization [WHO] (2020). Coronavirus Disease 2019 (COVID-19): Situation Report-51 (No. 51). (https://www.who.int/docs/default-source/ coronaviruse/situation-reports / 20200311-sitrep-51-covid19.pdf?sfvrsn=1ba62e57_10). Accessed 15 May 2020.

Xie X, Stone E, Zheng R, Zhang RG (2011). The 'Typhoon Eye Effect': determinants of distress during the SARS epidemic. Journal of Risk Research 14, 1091-1107.

\section{Appendix A}

Rapid Review ProtocolSearch Strategy Search strategies were developed using both keywords and MeSH terms. The search strategies were modified for each included database (PsycInfo and Medline). The * is a wildcard to search for terms that begin with the given string. Keywords and MeSH terms were searched for in title and abstract. Both databases were searched for papers between January 1985 to March 2020. Searches were restricted to English language papers only, in peer-reviewed journals. PsycInfo Search Strategy: PsycInfo was searched using the ProQuest interface on 06.04.2020 (temporal coverage from 1887- present). Search terms included: (Ab("young people" OR youth OR adolescen* OR "young adult" OR teen* OR child* OR youth OR "young person*" OR juvenile OR minors 
OR "emerging adult") OR (MJMAINSUBJECT. EXACT("Early Adolescence") OR MJMAINSUBJECT. EXACT("Emerging Adulthood"))) AND (ab(pandemic OR epidemic OR COVID-19 OR HIV/AIDS OR h1n1 OR MERS OR SARS OR ebola OR quarantin* OR "self isolation") OR (MJMAINSUBJECT.EXACT ("Pandemics") OR MJMAINSUBJECT.EXACT ("Epidemics") OR MAINSUBJECT.EXACT("HIV") OR MAINSUBJECT.EXACT(“AIDS") OR MAINSUBJECT. EXACT("Swine Influenza"))) AND (ab("mental health" OR "quality of life" OR "happiness with life" OR "life satisfaction" OR resilien* OR "depress*" OR "anxi" ${ }^{*}$ " OR "PTSD" OR "posttraumatic stress" OR loss OR bereavement OR grief OR psychological OR psychiatric OR insomnia OR psychosocial OR "key wellness" OR wellbeing) OR (MAINSUBJECT.EXACT ("Grief") OR MJMAINSUBJECT.EXACT("Depression (Emotion)") OR MAINSUBJECT.EXACT("Mental Health") OR MAINSUBJECT.EXACT(“Complex PTSD") OR MAINSUBJECT.EXACT("Major Depression") OR MAINSUBJECT.EXACT("Anxiety Disorders") OR MJMAINSUBJECT.EXACT ("Resilience (Psychological)"))) AND (la.exact ("ENG") AND PEER(yes)) Medline Search Strategy: Medline was searched using the ProQuest interface on 06.04.2020 (coverage from 1946 - present). Search terms included: ((ab("young people" OR youth OR adolescen* OR "young adult" OR teen* OR child* OR youth OR "young person*" OR juvenile OR minors OR "emerging adult") OR (MESH.EXACT ("Adolescent") OR MESH.EXACT("Young Adult") OR MESH.EXACT("Child"))) AND (ab(pandemic OR epidemic OR COVID-19 OR HIV/AIDS OR h1n1 OR MERS OR SARS OR ebola OR quarantin* OR "self isolation") OR (MESH.EXACT("HIV") OR MESH. EXACT("Epidemics") OR MESH.EXACT("Influenza A Virus, H1N1 Subtype") OR MESH. EXACT("Pandemics"))) AND (ab("mental health" OR "quality of life" OR "happiness with life" OR "life satisfaction" OR resilien* OR "depress" ${ }^{*}$ OR "anxi*" OR "PTSD" OR "posttraumatic stress" OR loss OR bereavement OR grief OR psychological OR psychiatric OR insomnia OR psychosocial OR "key wellness" OR wellbeing) OR (MESH.EXACT(“Mental Health") OR MESH.EXACT("Anxiety Disorders") OR MESH. EXACT("Bereavement") OR MESH.EXACT ("Resilience, Psychological") OR MESH.EXACT ("Grief") OR MESH.EXACT("Stress Disorders, Post-Traumatic")))) AND (la.exact("ENG") AND pd(19850101-20201231) AND PEER(yes)) Forward citations were searched using Google Scholar on 23.04.2020 and included/excluded for full-review based on the same inclusion/exclusion criteria as initial searches. Where full-texts could not be accessed following initial full-text screening, relevant authors were contacted. 